mRNA and serum creatinine-which were inversely correlated during acute rejectionwas more sensitive and specific for prediction of rejection reversal than either measurement alone. The level of FOXP3 mRNA in urine was inversely related to the time to onset of acute graft rejection following transplant $(P=0.01)$.

In summary, the levels of FOXP3 mRNA in urine independently predict outcome of acute allograft rejection; therefore, drugs that enhance the generation and function of regulatory $\mathrm{T}$ cells might improve rejection outcome in renal allograft recipients.

Kate Matthews

Original article Muthukumar T et al. (2005) Messenger RNA for FOXP3 in the urine of renal-allograft recipients. N Engl J Med 353: 2342-2351

\section{Meta-analysis of methods used to prevent post-transplantation CMV-related complications}

In a systematic review of data from randomized, controlled trials, researchers at the University of Nebraska Medical Center have compared two strategies for preventing CYTOMEGALOVIRUS (CMV) infection and its complications in solid organ transplant recipients.

The analysis included kidney and liver transplant recipients from 11 trials that assessed universal prophylaxis $(n=1,582)$ and 6 trials that evaluated a pre-emptive strategy $(n=398)$. Universal prophylaxis involved administration of aciclovir $\geq 2 \mathrm{~g} /$ day or ganciclovir $\geq 3 \mathrm{~g}$ /day for at least 60 days to all CMV seropositive patients and recipients of grafts from seropositive donors. The pre-emptive strategy involved at least 14 days of treatment for patients in whom CMV was detected during routine monitoring. Median follow-up was 6 months.

Both approaches significantly reduced the risk of developing CMV organ disease (defined as histological or clinical manifestation of CMV viremia) and acute allograft rejection, but only universal prophylaxis decreased bacterial and fungal infections and death $(P<0.05$ for all). Neither strategy reduced the risk of graft loss. There were insufficient data to assess CMV recurrence and resistance, or occurrence of late-onset CMV disease.

Subgroup analyses showed that aciclovir and ganciclovir were both effective in reducing CMV organ disease when used for universal prophylaxis.
This finding contradicts recent guidelines, which advise that only newer agents, such as ganciclovir and valganciclovir, be used in the solid organ transplant setting. The authors of this meta-analysis recommend that aciclovir remains an option for universal prophylaxis until a large, randomized, controlled trial resolves which anti-CMV drug is superior and most cost-effective.

Rachael Williams

Original article Kalil AC et al. (2005) Meta-analysis: the efficacy of strategies to prevent organ disease by cytomegalovirus in solid organ transplant recipients. Ann Intern Med 143: 870-880

\section{Minimizing post-transplantation weight gain would improve kidney graft survival}

A retrospective analysis of data from a university hospital in France has excluded METABOLIC SYNDROME as an independent determinant of renal transplantation outcome, but has revealed that substantial weight gain-which is common after transplantation-independently increases the risk of graft loss. Management of post-transplantation BMI should therefore be a priority.

One year following kidney transplantation, mean weight gain for the 292 graft recipients included in the study was $2.7 \pm 5.8 \mathrm{~kg}$. After adjusting for confounding variables, such as baseline BMI or metabolic syndrome, transplant recipients whose BMI increased by more than 5\% $(n=127)$ had a significantly augmented risk of graft loss (HAZARD RATIO 2.82, 95\% Cl 1.11-7.44, $P=0.015$ when censored for death). By contrast, there was no association between graft loss and metabolic syndrome, independent of the confounding effects of other factors such as serum C-REACTIVE PROTEIN level.

Interestingly, 1-year post-transplant weight gain in excess of $5 \%$ was less common in peritoneal dialysis patients than those on hemodialysis $(P=0.003)$. Ducloux et al. propose that higher LEPTIN and lower GHRELIN plasma levels in peritoneal dialysis patients reduced their appetite.

Attenuating weight gain using drugs or by regulating caloric intake might improve the outlook for kidney transplant recipients, but potential interventions first need to be tested in prospective trials.

Rachael Williams

Original article Ducloux D et al. (2005) One-year posttransplant weight gain is a risk factor for graft loss. Am J Transplant 5: 2922-2928

\section{GLOSSARY}

CYTOMEGALOVIRUS (CMV)

A herpes virus that is a common opportunistic infectious agent in immunosuppressed individuals such as solid organ transplantees and AIDS patients

\section{METABOLIC SYNDROME}

A collection of metabolic disorders that increase the risk of developing diabetes, heart disease or stroke; risk factors include high central obesity, high blood pressure and high insulin levels (Barker 1995 definition)

\section{BODY MASS INDEX (BMI)}

Ratio of body weight in kilograms to the square of height in meters; BMI > 28 or 27 for men and women, respectively, indicates obesity

\section{HAZARD RATIO}

The relative likelihood of experiencing a particular event; a hazard ratio of 0.5 indicates that one group has half the risk of the other group

\section{C-REACTIVE PROTEIN}

An indicator of acute inflammation

\section{LEPTIN}

A peptide of 167 amino acids that is released from fat and that produces inhibition of food intake and activation of the sympathetic nervous system

GHRELIN

Appetite-stimulating hormone produced by cells lining the stomach 